\title{
Comparisons of Serologic Responses of Early Syphilis to Treatment with a Single-Dose Benzathine Penicillin G Between HIV-Positive and HIV-Negative Patients
}

\author{
Kuan-Yin Lin · Chia-Jui Yang · Hsin-Yun Sun · Yu-Chung Chuang • \\ Lan-Hsin Chang $\cdot$ Wen-Chun Liu $\cdot$ Yi-Ching Su $\cdot$ Sui-Yuan Chang • \\ Chien-Ching Hung (i) $\cdot$ Shan-Chwen Chang
}

Received: March 14, 2021 / Accepted: April 19, 2021 / Published online: May 4, 2021

(C) The Author(s) 2021, corrected publication 2021

\begin{abstract}
Introduction: Poorer serologic responses of early syphilis to treatment have been inconsistently reported in HIV-positive patients compared with HIV-negative patients, but the interpretation of previous studies is limited by discrepant study designs. The present study
\end{abstract}

Supplementary Information The online version contains supplementary material available at https:// doi.org/10.1007/s40121-021-00450-6.

\section{K.-Y. Lin}

Department of Medicine, National Taiwan University Hospital Jin-Shan Branch, New Taipei City, Taiwan

\section{K.-Y. Lin}

Institute of Epidemiology and Preventive Medicine, College of Public Health, National Taiwan

University, Taipei, Taiwan

\section{C.-J. Yang}

Department of Internal Medicine, Far Eastern

Memorial Hospital, New Taipei City, Taiwan

\section{C.-J. Yang}

School of Medicine, National Yang-Ming

Chiao-Tung University, Taipei, Taiwan

H.-Y. Sun · Y.-C. Chuang · L.-H. Chang ·

W.-C. Liu - Y.-C. Su - C.-C. Hung ( $\bowtie) \cdot$ S.-C. Chang Department of Internal Medicine, National Taiwan University Hospital and National Taiwan University College of Medicine, 7 Chung-Shan South Road, Taipei, Taiwan e-mail: hcc0401@ntu.edu.tw aimed to evaluate the effect of HIV infection on the treatment response to a single dose of benzathine penicillin G (BPG) for early syphilis.

Methods: From January 2015 to March 2020, adult patients with early syphilis who received a single dose of BPG were enrolled and rapid plasma reagin (RPR) titers were periodically determined. The primary outcome was serologic response, defined as at least a fourfold decline of RPR titer at 12 months of BPG treatment compared with that at baseline, which was examined in the intention-to-treat (ITT) and per-

\author{
S.-Y. Chang \\ Department of Laboratory Medicine, National \\ Taiwan University Hospital and National Taiwan \\ University College of Medicine, Taipei, Taiwan \\ S.-Y. Chang \\ Department of Clinical Laboratory Sciences and \\ Medical Biotechnology, National Taiwan University \\ College of Medicine, Taipei, Taiwan \\ C.-C. Hung \\ Department of Tropical Medicine and Parasitology, \\ National Taiwan University College of Medicine, \\ Taipei, Taiwan \\ C.-C. Hung \\ Department of Medical Research, China Medical \\ University Hospital, Taichung, Taiwan \\ C.-C. Hung \\ China Medical University, Taichung, Taiwan
}


protocol analyses. Treatment failure included lack of at least a fourfold decline in RPR titers and at least a fourfold increase in RPR titers.

Results: We prospectively enrolled 184 HIVpositive and 68 HIV-negative participants with early syphilis, who were all men who have sex with men, with a higher proportion of previous syphilis (70.1\%) and early latent syphilis (64.1\%) among HIV-positive participants. In the ITT with last-observation-carried-forward analysis, HIV-positive participants had a significantly lower serologic response rate at 12 months of treatment than HIV-negative participants (73.4\% vs. 91.2\%). Of HIV-positive participants, $12.5 \%$ failed to achieve at least fourfold decline in RPR titers and $14.1 \%$ had at least a fourfold increase in RPR titers. The factors associated with 12-month serologic response were HIV infection (adjusted odds ratio $[\mathrm{AOR}]$ 0.33; 95\% confidence interval [CI] $0.13-0.81$ ) and RPR titer (per $1-\log _{2}$ increase, AOR 1.36; 95\% CI 1.23-1.51).

Conclusion: HIV-positive patients with early syphilis had poorer serologic responses to BPG treatment than HIV-negative patients during a 12-month follow-up period.

Keywords: Benzathine penicillin G; Rapid plasma reagin titer; Men who have sex with men; Recurrence; Reinfection; Jarisch-Herxheimer reaction

\section{Summary Points}

\section{Why carry out this study?}

Poorer serologic responses of early syphilis to single-dose benzathine penicillin (BPG) treatment have been inconsistently reported in HIV-positive patients compared with HIV-negative patients.

We compared the serologic responses to single-dose BPG treatment between HIVpositive and HIV-negative patients with early syphilis.

\section{What was learned from the study?}

Serologic responses of early syphilis to single-dose BPG treatment at 12 months were significantly poorer among HIVpositive patients $(73.4 \%)$ compared to HIV-negative patients (91.2\%).

The poorer responses were attributed to slower serologic responses and more treatment failures in HIV-positive patients.

Periodical serologic follow-up for syphilis is warranted among HIV-positive patients in the era of combination antiretroviral therapy scale-up and increasing rate of syphilis among at-risk populations.

\section{DIGITAL FEATURES}

This article is published with digital features, including a summary slide, to facilitate understanding of the article. To view digital features for this article go to https://doi.org/10.6084/ m9.figshare.14438996.

\section{INTRODUCTION}

The global burden of syphilis remains substantial despite effective antibiotic treatment being available for decades. The number of new cases of syphilis was around 6 million worldwide in 2016, with an estimated incidence of 1.6-1.7 cases per 1000 population [1]. An ever-growing epidemic of syphilis has been reported mainly among men who have sex with men (MSM), which is driven by online social networking, condomless sex, group sex, and recreational drug use [2]. Individuals receiving biomedical HIV treatment and prevention are particularly at high risk for syphilis [3, 4]. The surveillance data from the USA showed that primary and secondary syphilis were more prevalent among HIV-positive MSM (7.0\%) than among HIVnegative MSM (3.4\%) in 2018 [5]. In a longitudinal study to evaluate sexually transmitted diseases (STDs) among users of preexposure 
prophylaxis (PrEP) for HIV, the incidence of syphilis was 7.9 per 100 person-years after commencing PrEP [6].

The current STD treatment guidelines recommend single-dose benzathine penicillin G (BPG) for treating early syphilis regardless of HIV status [7]. However, the serologic responses of early syphilis to BPG treatment have been reportedly poorer among HIV-positive patients compared with their HIV-negative counterparts. For this reason, enhanced therapy with three weekly doses of BPG has been proposed to improve treatment outcome $[8,9]$. A systematic review that evaluated studies assessing serologic outcomes after syphilis treatment revealed an inconsistent relationship between HIV status and serologic responses [10]. Given the fact that these studies had differences in study populations and stages of syphilis, definitions used for serologic response, treatments administered, and follow-up frequencies and durations, the clinical uncertainty remains [11].

In the context of syphilis resurgence among individuals living with or at risk for HIV infection, further prospective studies with standard therapy and fixed follow-up time periods are needed to inform treatment recommendations. In this study, we aimed to compare the treatment responses to a single dose of BPG between HIV-positive and HIV-negative patients with early syphilis.

\section{METHODS}

\section{Setting and Study Participants}

This prospective observational study was conducted at the National Taiwan University Hospital, which is a major designated hospital for providing comprehensive health services for HIV testing, prevention, and treatment in Taiwan. From January 2015 to March 2020, HIVpositive and HIV-negative adult patients receiving a single dose of BPG for early syphilis were enrolled. While HIV-positive participants were recruited from the outpatient clinics, HIVnegative participants with syphilis were voluntary counseling and testing (VCT) clients [12].
The diagnosis of syphilis was made on the basis of a positive rapid plasma reagin (RPR) titer with a reactive Treponema pallidum particle agglutination (TPPA) assay. Early syphilis is divided into primary, secondary, and early latent stages, depending on clinical presentations [13]. Participants were classified to have primary syphilis if they presented with genital, anal, or oropharyngeal ulcers (chancre); participants with cutaneous rashes, mucosal lesions, or generalized lymphadenopathy were classified as having secondary syphilis; asymptomatic participants with reactive serologies were classified as early latent syphilis if they had documented negative RPR or at least fourfold increase of RPR titers within 12 months. Patients with RPR titers of less than 2 were excluded because RPR may remain reactive at a low titer even with BPG treatment [7]. Other exclusion criteria were having neurological manifestations, a history of penicillin allergy, no RPR titer available for assessment after treatment, and exposure to antibiotics with activity against $T$. pallidum, such as penicillins, ceftriaxone, doxycycline, or macrolides, within the preceding 4 weeks [14-16]. The study was approved by the Research Ethics Committee of National Taiwan University Hospital (registration number NTUH-201606110RINA) and the participants gave written informed consent to periodical follow-up serologic testing for syphilis and data collection before inclusion. The study was performed in accordance with the Helsinki Declaration of 1964 and its later amendments.

As a designated hospital in Taiwan, HIVpositive patients have been provided with freeof-charge combination antiretroviral therapy (cART), monitoring of CD4 cell count and plasma HIV RNA load (PVL), and serologies of viral hepatitis and syphilis at baseline and during follow-up. The national guidelines for HIV care recommend that non-treponemal serologic tests for syphilis should be performed at least annually and more frequently for at-risk patients; for those treated for syphilis, serologic follow-up should be performed every 3-6 months following treatment [7]. Individuals seeking VCT services have been offered free-ofcharge testing for HIV and syphilis. Those 
testing positive for HIV or syphilis are linked to medical care and receive treatment and further evaluation for viral hepatitis and other STDs [17].

\section{Treatment and Follow-up}

In accordance with current STDs treatment guidelines, the participants with early syphilis received a single intramuscular dose $(2.4$ million units) of BPG [7]. After administration of BPG, associated symptoms within $24 \mathrm{~h}$ were inquired by cell phone calls [18, 19]. Participants were followed up at weeks 1,2 , and 3, and months $1,2,3,6$ ( \pm 1 months), 9 ( \pm 1 months), and 12 ( \pm 3 months) from BPG treatment. At each follow-up visit, RPR titers were determined. To monitor HIV seroconversion among HIV-negative participants, testing for HIV was performed every 3 months.

Serologic response was defined as a decline of RPR titers by at least fourfold or reversion to nonreactivity compared with baseline RPR titers during the 12-month follow-up period. Treatment failure was defined as lack of at least a fourfold decline in RPR titers or at least a fourfold increase in RPR titers after achieving serologic response during follow-up $[8,9]$. The primary outcome was serologic response rate at 12 months from BPG treatment [7-9]. The secondary outcomes were serologic response rate at 6 months from BPG treatment, rates of treatment failure at 6 and 12 months, and Jarisch-Herxheimer $(\mathrm{JH})$ reaction following BPG injection. $\mathrm{JH}$ reaction was defined as the presence of fever (i.e., a temperature over $38.0^{\circ} \mathrm{C}$ ) and/or acute exacerbation of maculopapular skin rashes within $24 \mathrm{~h}$ after BPG injection $[18,19]$.

\section{Laboratory Investigations}

Serologic tests for syphilis included the RPR test (BD Macro-VueTMRPR Card tests) for screening and TPPA assay (FTI-SERODIA-TPPA; Fujirebio Taiwan Inc., Taoyuan, Taiwan) for confirmation. Blood samples obtained from VCT clients were tested for HIV with the use of particle agglutination (SFD HIV 1/2 PA; Bio-Rad FUJIREBIO, Tokyo, Japan) before December 2018 and fourth-generation antigen/antibody combination test (Alere Determine ${ }^{\mathrm{TM}}$ HIV-1/2 Ag/Ab Combo; Orgenics Ltd., Yavne, Israel) after January 2019. CD4 cell count was determined using flow cytometry (BD FACS Calibur; Becton Dickinson, CA, USA), and PVL was quantified using Cobas AmpliPrep/Cobas TaqMan HIV-1 test (version 2.0; Roche Molecular Systems) with a lower detection limit of 20 copies $/ \mathrm{ml}$. Hepatitis B virus surface antigen (HBsAg) and hepatitis $\mathrm{C}$ virus (HCV) antibody were determined with the use of an enzyme immunoassay (Abbott Laboratories, Abbott Park, IL).

\section{Statistical Analysis}

Statistical analyses were performed using STATA software version 12.0 (Stata Corporation, College Station, TX). Characteristics of the participants were compared between groups with the use of Fisher's exact test or chi-squared test for categorical variables and Wilcoxon-Mann-Whitney test for continuous variables. Serologic response was estimated in the intention-to-treat (ITT) and per-protocol (PP) analyses. For participants with missing data on RPR titers and other time-updated variables at 6 and 12 months in the ITT analysis, the lastobservation-carried-forward (LOCF) approach was applied to impute missing data. The associations between time-updated variables and outcome measures were identified using the generalized estimating equations in a logistic regression model. Considering that participants could be reinfected even following successful treatment, time to serologic response was assessed with the Kaplan-Meier method and Cox proportional hazards regression analysis was used to estimate the predictors of achieving serologic response. Variables with $P$ values of less than 0.1 in the univariate analysis were selected for entry into the multivariate model, and a backward selection process was used to determine the final model. The 95\% confidence intervals (CIs) of odds ratios or hazard ratios were reported to estimate the effects of covariates. All tests were two-tailed and a $P$ value of less than 0.05 was considered statistically significant. 
Table 1 Baseline characteristics of participants with early syphilis who received a single dose of BPG

\begin{tabular}{|c|c|c|c|}
\hline Variable & $\begin{array}{l}\text { HIV-positive participants } \\
(N=184)\end{array}$ & $\begin{array}{l}\text { HIV-negative participants } \\
(N=68)\end{array}$ & $P$ \\
\hline Age, median (IQR), years & $36(31-42)$ & $31(25-35)$ & $<0.001$ \\
\hline MSM, $n(\%)$ & $184(100)$ & $68(100)$ & 0.999 \\
\hline HBsAg positivity, $n$ (\%) & $28(15.2)$ & $2 / 38(5.3)$ & 0.102 \\
\hline Anti-HCV positivity, $n(\%)$ & $22(12.0)$ & $2 / 47(4.3)$ & 0.123 \\
\hline Previous history of syphilis, $n$ (\%) & $129(70.1)$ & $14(20.6)$ & $<0.001$ \\
\hline \multicolumn{4}{|l|}{ Syphilis stage, $n(\%)$} \\
\hline Primary & $13(7.1)$ & $8(11.8)$ & 0.231 \\
\hline Secondary & $53(28.8)$ & $31^{a}(45.6)$ & 0.012 \\
\hline Early latent & $118(64.1)$ & $33(48.5)$ & 0.025 \\
\hline RPR titer, median (IQR) & $1: 128(1: 64-1: 512)$ & $1: 128(1: 48-1: 256)$ & 0.330 \\
\hline Receiving cART at baseline, $n(\%)$ & $170(92.4)$ & NA & - \\
\hline $\begin{array}{l}\mathrm{CD} 4 \text { cell count at baseline, median (IQR), } \\
\text { cells } / \mathrm{mm}^{3}\end{array}$ & $577(453-759)$ & NA & - \\
\hline$<200, n(\%)$ & $6(3.3)$ & NA & - \\
\hline$>350, n(\%)$ & $161(87.5)$ & NA & - \\
\hline $\begin{array}{l}\text { PVL at baseline, median (range), } \log _{10} \text { copies/ } \\
\mathrm{mL}\end{array}$ & UD (UD-6.36) & NA & - \\
\hline$<200$ copies/mL, $n(\%)$ & $169(91.8)$ & NA & - \\
\hline Receiving cART at 12 months, $n$ (\%) & $183(99.5)$ & NA & - \\
\hline $\begin{array}{l}\text { CD } 4 \text { cell count at } 12 \text { months, median (IQR), } \\
\text { cells } / \mathrm{mm}^{3}\end{array}$ & $628(510-794)$ & NA & - \\
\hline$<200, n(\%)$ & $1(0.5)$ & NA & - \\
\hline$>350, n(\%)$ & $172(93.5)$ & NA & - \\
\hline $\begin{array}{l}\text { PVL at } 12 \text { months, median (range), } \log _{10} \\
\text { copies } / \mathrm{mL}\end{array}$ & UD (UD-5.50) & NA & - \\
\hline$<200$ copies/mL, $n(\%)$ & $182(98.9)$ & NA & - \\
\hline
\end{tabular}

$B P G$ benzathine penicillin G, $c A R T$ combination antiretroviral therapy, $H B s A g$ hepatitis B surface antigen, $H C V$ hepatitis $\mathrm{C}$ virus, $I Q R$ interquartile range, $M S M$ men who have sex with men, $P V L$ plasma HIV RNA load, $R P R$ rapid plasma regain, $U D$ undetectable

${ }^{a}$ Four HIV-negative participants had concurrent chancres and manifestations of secondary syphilis 


\section{RESULTS}

\section{Clinical Characteristics of Participants}

During the study period, we enrolled 184 HIVpositive and $68 \mathrm{HIV}$-negative participants who received a single-dose BPG for early syphilis. Table 1 shows the baseline characteristics of the participants. All participants had completed 12-month follow-up. The participants were all MSM with a median age of 34 years. HIV-positive participants had a cART coverage rate of $92.4 \%$, with a median baseline CD4 count of 577 cells $/ \mathrm{mm}^{3}$ and $91.8 \%$ having achieved PVL $<200$ copies/mL. Compared with HIVnegative participants, HIV-positive participants were older (median age 36 vs. 31 years) and had higher rates of hepatitis B virus (HBV) and HCV coinfections $(15.2 \%$ vs. $5.3 \%$ and $12.0 \%$ vs. $4.3 \%$, respectively) and previous history of syphilis (70.1\% vs. $20.6 \%$ ). More HIV-positive participants presented with early latent syphilis (64.1\%) than secondary syphilis (28.8\%), whereas similar proportions of HIV-negative participants presented with secondary syphilis (45.6\%) and early latent syphilis (48.5\%). The baseline median RPR titer was 1:128, without significant difference between HIV-positive and HIV-negative participants.

\section{Serologic Responses After BPG Treatment}

In the ITT with LOCF analysis, HIV-positive participants had statistically significantly lower serologic response rates at 6 and 12 months of BPG treatment $(74.5 \%$ [137/184] and 73.4\% [135/184], respectively) compared with those among HIV-negative participants (89.7\% [61/ 68] and 91.2\% [62/68], respectively) $(P<0.05)$ (Fig. 1). For 26 and 33 participants with missing data on RPR titers at 6 and 12 months, respectively, the data at months 3 and 6-9 were mostly carried forward. In the PP analysis, HIVpositive participants achieved significantly slower serologic response than HIV-negative participants (Fig. S1): $33.8 \%$ vs. $64.3 \%$ at month $1 \quad(P<0.001), \quad 63.3 \%$ vs. $90.2 \%$ at month $2 \quad(P<0.001), \quad 62.3 \%$ vs. $90.0 \%$ at month $3 \quad(P<0.001), \quad 76.1 \%$ vs. $92.0 \%$ at month $6(P=0.024)$, and $73.0 \%$ vs. $88.9 \%$ at month $12(P=0.042)$ following BPG treatment. While more than $90 \%$ of HIV-positive participants had RPR follow-ups at months 6 and 12, the proportion of HIV-negative participants with available RPR titers was $73.5 \%$ at month 6 , which decreased to $66.2 \%$ at month 12 (Fig. S1). Five participants had HIV seroconversion during the follow-up period: five at 6-month follow-up, two at 9-month follow-up, and one at

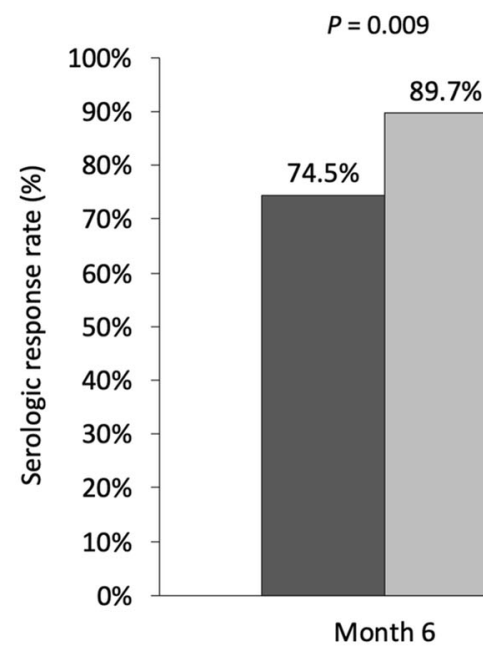

Fig. 1 Serologic response rates among HIV-positive and HIV-negative participants at 6 and 12 months of BPG treatment in the ITT with LOCF analysis. BPG

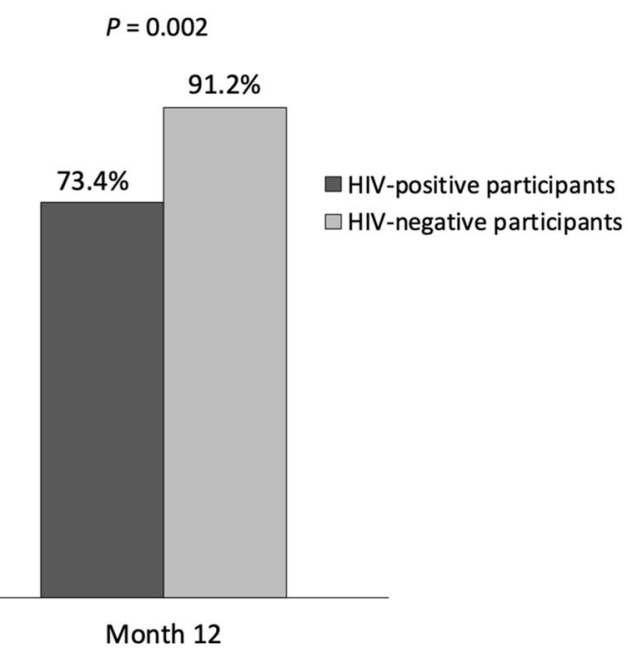

benzathine penicillin G, ITT intention-to-treat, LOCF last-observation-carried-forward 


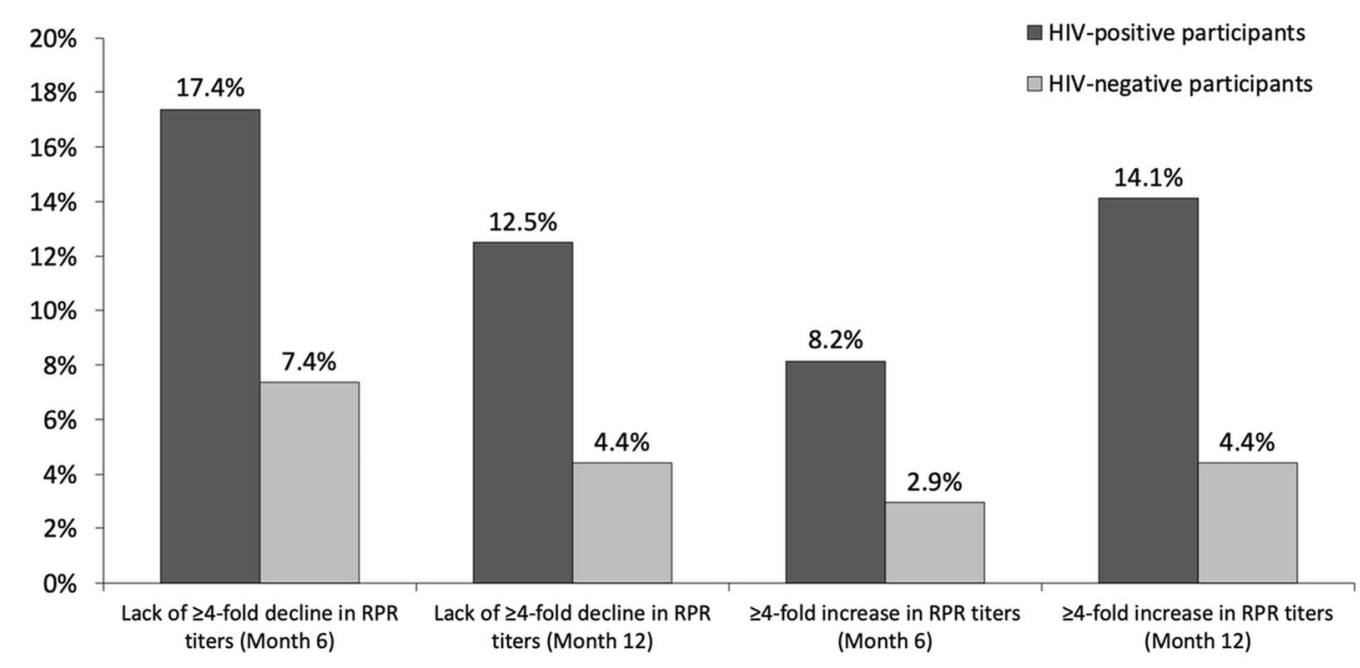

Fig. 2 Reasons for the failure of achieving serologic response among HIV-positive and HIV-negative participants at 6 and 12 months of BPG treatment in the ITT

12-month follow-up; all of them achieved serologic response of early syphilis at month 12 .

\section{Reasons for Treatment Failure}

A total of 47 (25.5\%) HIV-positive and 7 (10.3\%) HIV-negative participants experienced treatment failure at 6 months of BPG treatment, whereas 49 (26.6\%) HIV-positive and 6 (8.8\%) HIV-negative participants had treatment failure at 12 months. The reasons for treatment failure in the ITT with LOCF analysis are depicted in Fig. 2. Of HIV-positive participants, the rates of lack of at least a fourfold decline in RPR titers and at least a fourfold increase in RPR titers at month 6 were $17.4 \%(32 / 184)$ and $8.2 \%(15 /$ $184)$, respectively; at month 12 , the rate of lack of at least a fourfold decline in RPR titers decreased to $12.5 \%(23 / 184)$ while the rate of at least a fourfold increase in RPR titers increased to $14.1 \%(26 / 184)$. Of HIV-negative participants, the rates of lack of at least a fourfold decline in RPR titers and at least a fourfold increase in RPR titers at month 6 were $7.4 \%$ (5/ 68 ) and $2.9 \%$ (2/68), respectively; at month 12 , the rates of lack of at least a fourfold decline in RPR titers $(3 / 68,4.4 \%)$ and at least a fourfold increase in RPR titers $(3 / 68,4.4 \%)$ were similar. with LOCF analysis. BPG benzathine penicillin G, ITT intention-to-treat, LOCT last-observation-carried-forward

\section{Factors Associated with Serologic Response}

The factors associated with serologic response at month 12 are shown in Table 2 . In univariate analysis, HIV seronegativity, HCV seropositivity, and higher RPR titers were positively associated with 12-month serologic response. In multivariate analysis, the independent factors associated with 12-month serologic response included HIV infection (adjusted odds ratio [AOR] 0.33; 95\% CI 0.13-0.81) and RPR titer (per 1- $\log _{2}$ increase, AOR 1.36; 95\% CI 1.23-1.51) (Table 2). Among HIV-positive participants, 12-month serologic response was not associated with cART, CD4 count, and PVL suppression.

Time to serologic response after BPG treatment was compared between HIV-positive and HIV-negative participants with the use of the Kaplan-Meier method (Fig. S2). Of the participants, 168 (91.3\%) HIV-positive and 64 (94.1\%) HIV-negative participants ever achieved serologic response during follow-up $(P=0.638)$. The median time to serologic response was significantly longer in the HIV-positive group (58 days; IQR 35-127) than the HIV-negative group (46 days; IQR 31-69) (log-rank test, $P=0.016)$. After adjustment for potential confounders, HIV infection remained an 
Table 2 Logistic regression analysis for factors associated with serologic response at 12 months of BPG treatment

\begin{tabular}{|c|c|c|c|c|}
\hline & \multicolumn{2}{|c|}{ Univariate analysis } & \multicolumn{2}{|c|}{ Multivariate analysis } \\
\hline & OR (95\% CI) & $P$ & $\mathrm{OR}^{\mathrm{a}}(95 \% \mathrm{CI})$ & $P$ \\
\hline \multicolumn{5}{|l|}{ Baseline characteristics } \\
\hline HIV infection at each visit & $0.27(0.11-0.64)$ & 0.003 & $0.33(0.13-0.81)$ & 0.015 \\
\hline Age, per 1-year increase & $0.98(0.96-1.00)$ & 0.111 & & \\
\hline HBsAg positivity & $0.88(0.53-1.47)$ & 0.622 & & \\
\hline Anti-HCV positivity & $2.40(1.16-4.97)$ & 0.018 & $1.94(0.92-4.10)$ & 0.082 \\
\hline Previous history of syphilis & $0.79(0.53-1.16)$ & 0.224 & & \\
\hline \multicolumn{5}{|l|}{ Syphilis stage } \\
\hline Primary & $1.00(0.51-1.96)$ & 0.993 & & \\
\hline Secondary & $1.32(0.88-1.99)$ & 0.178 & & \\
\hline Early latent & $0.91(0.62-1.32)$ & 0.607 & & \\
\hline RPR titer, per $1-\log _{2}$ increase & $1.36(1.23-1.50)$ & $<0.001$ & $1.36(1.23-1.51)$ & $<0.001$ \\
\hline Receiving cART at each visit & $0.27(0.06-1.17)$ & 0.080 & & \\
\hline Time-updated CD4 cell count, per 10 -cell $/ \mathrm{mm}^{3}$ increase & $0.99(0.99-1.00)$ & 0.034 & & \\
\hline Time-updated PVL $<200$ copies $/ \mathrm{mL}$ & $0.47(0.16-1.39)$ & 0.172 & & \\
\hline
\end{tabular}

$B P G$ benzathine penicillin G, $C A R T$ combination antiretroviral therapy, GEE generalized estimating equations, $H B s A g$ hepatitis B surface antigen, $H C V$ hepatitis $\mathrm{C}$ virus, $O R$ odds ratio, $P V L$ plasma HIV RNA load, RPR rapid plasma regain

${ }^{a}$ The ORs are the estimates of the effect of time-updated covariates on serologic response at 12 months of BPG treatment, adjusted for baseline HIV status, anti-HCV positivity, and RPR titers using GEE method in a logistic regression model. Receipt of cART and CD4 cell count, variables collinear with HIV infection, was not included in the multivariate model

independent factor associated with a reduced likelihood of achieving serologic response (adjusted hazard ratio [AHR] 0.72; 95\% CI 0.51-0.99) (Table S1).

\section{Drug Reactions to BPG Treatment}

The overall rate of drug reactions following BPG treatment was $35.3 \%$ and $\mathrm{JH}$ reaction accounted for $82.0 \%$ of the reactions (Table S2). The median time to developing $\mathrm{JH}$ reaction was $5 \mathrm{~h}$ (interquartile range $4-7 \mathrm{~h}$ ). The most common symptom was fever $(20.6 \%)$, followed by exacerbation of maculopapular skin rashes (15.9\%) and lassitude (9.5\%). Compared with HIV-negative participants, HIV-positive participants had significant lower rates of drug reactions $(29.9 \%$ vs. $50.0 \%)$, JH reaction $(25.0 \%$ vs. $39.7 \%)$, lassitude (5.4\% vs. $20.6 \%$ ), and itchiness (0\% vs. $4.4 \%)$. After adjustment for covariates in multivariate analysis, secondary syphilis was independently associated with $\mathrm{JH}$ reaction (AOR 3.90; 95\% CI 2.02-7.52) (Table S3).

\section{DISCUSSION}

In this study with periodical serologic follow-up after treatment of syphilis, we found that serologic responses of early syphilis to single-dose BPG treatment at 12 months were significantly poorer among HIV-positive patients (73.4\%) compared to HIV-negative patients (91.2\%). HIV-positive patients not only had delayed serologic responses to BPG treatment but also experienced more treatment failures. While RPR 
titer was positively associated with serologic response, higher CD4 counts and viral suppression had no impact on serologic responses among the HIV-positive patients. JH reaction following administration of BPG treatment was not uncommon $(29.0 \%)$, with a positive correlation with secondary syphilis.

Only few studies prospectively evaluated the association of HIV status with treatment response of syphilis. In a randomized trial enrolling 101 HIV-positive and $440 \mathrm{HIV}$-negative patients, frequent follow-up testing was performed at 2 weeks and 1, 2, 3, 6, 9, and 12 months to clarify the effects of HIV status and BPG treatment enhanced with amoxicillin and probenecid on early syphilis. The study showed that HIV-positive patients were more likely to experience serologic failure than HIVnegative patients at 12 months: $14 \%$ vs. $8 \%$ in patients of the primary stage and $19 \%$ vs. $6 \%$ in those of the secondary stage [20]. Our study with a similar follow-up frequency also demonstrated similar serologic responses. In another randomized trial to compare the treatment responses between a single dose of BPG and azithromycin for early syphilis, $171 \mathrm{HIV}$ positive and 157 HIV-negative patients were followed up on a 3-month basis. The cure rate among HIV-positive patients was $94.7 \%$ at 9 months, which was slightly lower than that among HIV-negative patients (98.4\%) but without statistically significant difference [21]. Notably, patients in this trial stopped participation once cured, and thus reinfection could not be identified. Our study also showed similarly high rates (greater than 90\%) of patients having ever achieved serologic response in both groups. Therefore, the lower serologic response in the HIV-positive group may be driven in part by more reinfections [8].

Studies retrospectively evaluating the association of HIV status with serologic outcomes of syphilis included patients with varied risk behaviors and syphilis stages, different treatments and definitions of serologic responses used, and different follow-up frequencies and durations [22-25]. The study heterogeneity limits the interpretation of published data, especially the discrepancies between irregular follow-up frequencies and outcomes leading to biased results $[23,26]$. A large retrospective analysis of 1321 patients from two HIVserodiscordant couple cohorts reported that $67 \%$ had responses to BPG treatment, $26.6 \%$ had serofast responses, and $6.5 \%$ became reinfected by 400 days. No significant differences were found between HIV-positive and HIVnegative patients in the multivariate analysis and Cox proportional hazards model. Nevertheless, the study included both incident and prevalent syphilis cases, among whom several patients were treated with two or three weekly doses of BPG treatment [27]. In contrast, other studies stratifying data by syphilis stages demonstrated that HIV-positive patients with primary or early syphilis had slower serologic responses compared with HIV-negative patients $[23,25]$. Through periodical serologic follow-up in our study, the significant differences in serologic responses were also observed between the two groups from month 1 . Therefore, the lower serologic response in the HIV-positive group may also be attributed to slower treatment response.

In addition to HIV status, previous studies also identified the correlation between serologic response and younger age, history of prior syphilis, higher baseline non-treponemal titers, and earlier syphilis stage [10]. Of these, the effects of baseline RPR titers as well as primary or secondary stage on serologic response were consistently demonstrated in studies conducted in the HIV-positive population $[8,28,29]$. Since immunosuppression is regarded as a predictor of serologic failure, the impact of CD4 count, PVL, and cART use on serologic response of syphilis among HIV-positive patients has been examined [30]. A study assessing HIV-positive patients with a median CD4 count of 280 cells/ $\mathrm{mm}^{3}$ from 1990 to 2006 found that CD4 count below 200 cells $/ \mathrm{mm}^{3}$ increased the risk of serologic failure and receipt of cART reduced the rate of serologic failure by $60 \%$ [31]. In the era of cART scale-up, most studies included HIVpositive patients with higher median CD4 counts (greater than 450 cells $/ \mathrm{mm}^{3}$ ) and found no associations of CD4 count, PVL, and cART use with treatment responses $[8,16,29]$. In line with previous studies, our study including patients with a high cART coverage and median 
CD4 count did not find the association of immune status with serologic responses to BPG.

To improve serologic responses to BPG treatment among HIV-positive patients, enhanced therapy with three weekly doses of BPG has been proposed. A prospective study of HIV-positive patients with early syphilis failed to demonstrate the non-inferiority of a singledose regimen to a three-weekly regimen $(67.1 \%$ vs. $74.8 \%$ ) at 12 months. The effectiveness in the study was underestimated because of a substantial rate of reinfections, and there was no difference in serologic response $(88.1 \%$ vs. $86.0 \%$ ) when evaluating the proportions of patients ever achieving at least a fourfold decline in RPR titers after treatment [8]. Another randomized trial with a small sample size also showed similar serologic outcomes in HIV-positive patients receiving single-dose and three-weekly regimens for early syphilis [9]. Therefore, the current evidence supporting single-dose BPG treatment for early syphilis is limited by study designs. Our study showed that HIV-positive patients had slower serologic responses and more treatment failures after excluding those who might have been reinfected with syphilis, suggesting that further well-designed randomized trials are warranted to examine if enhanced treatment is needed to improve the treatment response.

Our prospective study administered a single dose of BPG as standard therapy for early syphilis and performed periodical follow-up to monitor serologic response. A strength of our study is that it improves our understanding of differences in treatment responses between HIV-positive and HIV-negative patients. However, our study has several limitations. First, the baseline characteristics across study groups were not balanced; nevertheless, HIV infection was still an independent factor associated with achieving serologic response after adjusting for covariates in both logistic and Cox regression analyses. Second, reinfection and relapse episodes may not be captured because of the lower retention rate among VCT clients. Nevertheless, PP analysis from periodical follow-up testing could help estimate serologic responses. The proportion of patients with available RPR data at 1 month of BPG treatment was similar between the two groups, and serologic responses were significantly higher among HIV-negative VCT clients. Third, the lack of detailed information on exposure behaviors precluded us from distinguishing patients with reinfections from those with serologic failures. Therefore, the objective criteria of serologic patterns over time were used to differentiate treatment failures.

\section{CONCLUSIONS}

We found that HIV-positive patients had slower serologic responses and more treatment failures than HIV-negative patients after a single-dose BPG for early syphilis, which contributed to poorer serologic responses observed during a 12-month follow-up period. Our results support that, in the era of cART scale-up and increasing rate of syphilis among at-risk populations, periodical serologic follow-up for syphilis is warranted among HIV-positive patients.

\section{ACKNOWLEDGEMENTS}

We would like to thank the participants of the study.

Funding. No financial support or grants were received for this study. The Rapid Service Fee was funded by the authors.

Authorship. All named authors meet the International Committee of Medical Journal Editors (ICMJE) criteria for authorship for this article, take responsibility for the integrity of the work as a whole, and have given their approval for this version to be published.

Authorship Contributions. Chien-Ching Hung, Sui-Yuan Chang, and Shan-Chwen Chang managed and supervised the study. Kuan-Yin Lin, Chia-Jui Yang, and Chien-Ching Hung contributed to the study concept and design. Lan-Hsin Chang, Yi-Ching Su, and SuiYuan Chang performed the experiments. KuanYin Lin, Hsin-Yun Sun, Yu-Chung Chuang, LanHsin Chang, Wen-Chun Liu, Yi-Ching Su, and 
Chien-Ching Hung were involved in collection and assembly of clinical data. Kuan-Yin Lin, Chia-Jui Yang, Yi-Ching Su, Sui-Yuan Chang, and Chien-Ching Hung participated in data analysis. Kuan-Yin Lin, Chia-Jui Yang, ChienChing Hung, and Shan-Chwen Chang undertook interpretation of the data and drafted the report. All authors reviewed and approved the final version of the report.

Disclosures. Kuan-Yin Lin, Chia-Jui Yang, Hsin-Yun Sun, Yu-Chung Chuang, Lan-Hsin Chang, Wen-Chun Liu, Yi-Ching Su, Sui-Yuan Chang, Chien-Ching Hung and Shan-Chwen Chang have nothing to disclose.

Compliance with Ethics Guidelines. The study was approved by the Research Ethics Committee of National Taiwan University Hospital (registration number, NTUH201606110RINA) and the participants gave written informed consent to periodical followup serologic testing for syphilis and data collection before inclusion. The study was performed in accordance with the Helsinki Declaration of 1964 and its later amendments.

Data Availability. The datasets generated during and analyzed during the current study are available from the corresponding author on reasonable request.

Open Access. This article is licensed under a Creative Commons Attribution-NonCommercial 4.0 International License, which permits any non-commercial use, sharing, adaptation, distribution and reproduction in any medium or format, as long as you give appropriate credit to the original author(s) and the source, provide a link to the Creative Commons licence, and indicate if changes were made. The images or other third party material in this article are included in the article's Creative Commons licence, unless indicated otherwise in a credit line to the material. If material is not included in the article's Creative Commons licence and your intended use is not permitted by statutory regulation or exceeds the permitted use, you will need to obtain permission directly from the copyright holder. To view a copy of this licence, visit http:// creativecommons.org/licenses/by-nc/4.0/.

\section{REFERENCES}

1. World Health Organization. Report on global sexually transmitted infection surveillance, 2018. https://www.who.int/reproductivehealth/ publications/stis-surveillance-2018/en/. Accessed 10 Mar 2021.

2. Chow EPF, Grulich AE, Fairley CK. Epidemiology and prevention of sexually transmitted infections in men who have sex with men at risk of HIV. Lancet HIV. 2019;6(6):e396-405.

3. Lin KY, Sun HY, Lee TF, et al. High prevalence of sexually transmitted coinfections among at-risk people living with HIV. J Formos Med Assoc. 2020;S0929-6646(20):30608-12.

4. Fields SD, Tung E. Patient-focused selection of PrEP medication for individuals at risk of HIV: a narrative review. Infect Dis Ther. 2021;10(1):165-86.

5. Centers for Disease Control and Prevention. Sexually transmitted disease surveillance 2018. https:// www.cdc.gov/std/stats18/STDSurveillance2018full-report.pdf. Accessed 10 Mar 2021.

6. Traeger MW, Cornelisse VJ, Asselin J, et al. Association of HIV preexposure prophylaxis with incidence of sexually transmitted infections among individuals at high risk of HIV infection. JAMA. 2019;321(14):1380-90.

7. Workowski KA, Bolan GA. Sexually transmitted diseases treatment guidelines, 2015. MMWR Recomm Rep. 2015;64(RR-03):1-137.

8. Yang CJ, Lee NY, Chen TC, et al. One dose versus three weekly doses of benzathine penicillin $G$ for patients co-infected with HIV and early syphilis: a multicenter, prospective observational study. PLoS One. 2014;9(10):e109667.

9. Andrade R, Rodriguez-Barradas MC, Yasukawa K, Villarreal E, Ross M, Serpa JA. Single dose versus 3 doses of intramuscular benzathine penicillin for early syphilis in HIV: a randomized clinical trial. Clin Infect Dis. 2017;64(6):759-64.

10. Seña AC, Zhang XH, Li T, et al. A systematic review of syphilis serological treatment outcomes in HIVinfected and HIV-uninfected persons: rethinking the significance of serological non-responsiveness and the serofast state after therapy. BMC Infect Dis. $2015 ; 15: 479$. 
11. Marra CM, Ghanem KG. Centers for Disease Control and Prevention syphilis summit: difficult clinical and patient management issues. Sex Transm Dis. 2018;45(9S Suppl 1):S10-2.

12. Lee YC, Liu WC, Hsieh YL, et al. Non-opioid recreational drug use and a prolonged HIV outbreak among men who have sex with men in Taiwan: an incident case-control study, 2006-2015. J Formos Med Assoc. 2021;S0929-6646(21):00120.

13. Ghanem KG, Ram S, Rice PA. The modern epidemic of syphilis. N Engl J Med. 2020;382(9):845-54.

14. Wu BR, Yang CJ, Tsai MS, et al. Multicentre surveillance of prevalence of the 23S rRNA A2058G and $\mathrm{A} 2059 \mathrm{G}$ point mutations and molecular subtypes of Treponema pallidum in Taiwan, 2009-2013. Clin Microbiol Infect. 2014;20(8):802-7.

15. Yang CJ, Tang HJ, Chang SY, et al. Comparison of serological responses to single-dose azithromycin (2 g) versus benzathine penicillin $G$ in the treatment of early syphilis in HIV-infected patients in an area of low prevalence of macrolide-resistant Treponema pallidum infection. J Antimicrob Chemother. 2016;71(3):775-82.

16. Tsai JC, Lin YH, Lu PL, et al. Comparison of serological response to doxycycline versus benzathine penicillin $G$ in the treatment of early syphilis in HIV-infected patients: a multi-center observational study. PLoS One. 2014;9(10):e109813.

17. Huang YC, Sun HY, Chuang YC, et al. Short-term outcomes of rapid initiation of antiretroviral therapy among HIV-positive patients: real-world experience from a single-centre retrospective cohort in Taiwan. BMJ Open. 2019;9(9):e033246.

18. Yang CJ, Lee NY, Lin YH, et al. Jarisch-Herxheimer reaction after penicillin therapy among patients with syphilis in the era of the HIV infection epidemic: incidence and risk factors. Clin Infect Dis. 2010;51(8):976-9.

19. Tsai MS, Yang CJ, Lee NY, et al. Jarisch-Herxheimer reaction among HIV-positive patients with early syphilis: azithromycin versus benzathine penicillin G therapy. J Int AIDS Soc. 2014;17(1):18993.

20. Rolfs RT, Joesoef MR, Hendershot EF, et al. A randomized trial of enhanced therapy for early syphilis in patients with and without human immunodeficiency virus infection. The Syphilis and HIV Study Group. N Engl J Med. 1997;337(5):307-14.

21. Riedner G, Rusizoka M, Todd J, et al. Single-dose azithromycin versus penicillin $\mathrm{G}$ benzathine for the treatment of early syphilis. $\mathrm{N}$ Engl $\mathrm{J}$ Med. 2005;353(12):1236-44.

22. Long CM, Klausner JD, Leon S, et al. Syphilis treatment and HIV infection in a population-based study of persons at high risk for sexually transmitted disease/HIV infection in Lima. Peru Sex Transm Dis. 2006;33(3):151-5.

23. Ghanem KG, Erbelding EJ, Wiener ZS, Rompalo AM. Serological response to syphilis treatment in HIV-positive and HIV-negative patients attending sexually transmitted diseases clinics. Sex Transm Dis. 2007;83(2):97-101.

24. Manavi K, McMillan A. The outcome of treatment of early latent syphilis and syphilis with undetermined duration in HIV-infected and HIV-uninfected patients. Int J STD AIDS. 2007;18(12):814-8.

25. Knaute DF, Graf N, Lautenschlager S, Weber R, Bosshard PP. Serological response to treatment of syphilis according to disease stage and HIV status. Clin Infect Dis. 2012;55(12):1615-22.

26. Atsawawaranunt K, Kittiyaowamarn R, Phonrat B, Kamolratanakul S, Kangvalpornroj T, Dhitavat J. Time to serological cure and associated factors among syphilis patients with and without HIV in a sexually transmitted infections center, Thailand. Sex Transm Dis. 2020;47(5):283-9.

27. Dionne-Odom J, Karita E, Kilembe W, et al. Syphilis treatment response among HIV-discordant couples in Zambia and Rwanda. Clin Infect Dis. 2013;56(12):1829-37.

28. Jinno S, Anker B, Kaur P, Bristow CC, Klausner JD. Predictors of serological failure after treatment in HIV-infected patients with early syphilis in the emerging era of universal antiretroviral therapy use. BMC Infects Dis. 2013;13:605.

29. Wu BR, Tsai MS, Yang CJ, et al. Spirochetemia due to Treponema pallidum using polymerase-chain-reaction assays in patients with early syphilis: prevalence, associated factors and treatment response. Clin Microbiol Infect. 2014;20(8):O524-7.

30. Spagnuolo V, Poli A, Galli L, et al. Incidence and predictors of serological treatment response in early and late syphilis among people living with HIV. Open Forum Infect Dis. 2019;6(1):ofy324.

31. Ghanem KG, Moore RD, Rompalo AM, Erbelding EJ, Zenilman JM, Gebo KA. Antiretroviral therapy is associated with reduced serologic failure rates for syphilis among HIV-infected patients. Clin Infect Dis. 2008;47(2):258-65. 